\title{
HYPONORMAL MATRICES AND SEMIDEFINITE INVARIANT SUBSPACES IN INDEFINITE INNER PRODUCTS*
}

\author{
CHRISTIAN MEHL ${ }^{\dagger}$, ANDRÉ C. M. RAN $\ddagger$, AND LEIBA RODMAN§
}

\begin{abstract}
It is shown that, for any given polynomially normal matrix with respect to an indefinite inner product, a nonnegative (with respect to the indefinite inner product) invariant subspace always admits an extension to an invariant maximal nonnegative subspace. Such an extension property is known to hold true for general normal matrices if the nonnegative invariant subspace is actually neutral. An example is constructed showing that the extension property does not generally hold true for normal matrices, even when the nonnegative invariant subspace is assumed to be positive. On the other hand, it is proved that the extension property holds true for hyponormal (with respect to the indefinite inner product) matrices under certain additional hypotheses.
\end{abstract}

Key words. Indefinite inner products, Semidefinite invariant subspaces, Hyponormal matrices, Normal matrices.

AMS subject classifications. 15A63, 15A57.

1. Introduction. Let $\mathbb{C}^{n}$ be the vector space of $n$-dimensional columns with complex components. We often write $Y \geq 0$ to indicate that a complex matrix $Y$ is positive semidefinite. Fix the indefinite inner product $[\cdot, \cdot]$ determined by an invertible Hermitian $n \times n$ matrix $H$ via the formula

$$
[x, y]=\langle H x, y\rangle, \quad x, y \in \mathbb{C}^{n} .
$$

(Here, $\langle\cdot, \cdot\rangle$ denotes the standard inner product in $\mathbb{C}^{n}$.) A subspace $\mathcal{M} \subseteq \mathbb{C}^{n}$ is said to be $H$-nonnegative if $[x, x] \geq 0$ for every $x \in \mathcal{M}, H$-positive if $[x, x]>0$ for every nonzero $x \in \mathcal{M}, H$-nonpositive if $[x, x] \leq 0$ for every $x \in \mathcal{M}, H$-negative if $[x, x]<0$ for every nonzero $x \in \mathcal{M}$, and $H$-neutral if $[x, x]=0$ for every $x \in \mathcal{M}$. Note that by default the zero subspace is $H$-positive as well as $H$-negative. An $H$-nonnegative subspace is said to be maximal $H$-nonnegative if it is not properly contained in any larger $H$-nonnegative subspace. It is easy to see that an $H$-nonnegative subspace is maximal if and only if its dimension is equal to the number of positive eigenvalues of $H$ (counted with multiplicities).

Let $X^{[*]}:=H^{-1} X^{*} H$ denote the adjoint of a matrix $X \in \mathbb{C}^{n \times n}$ with respect to the indefinite inner product, i.e., $X^{[*]}$ is the unique matrix satisfying $[x, X y]=$

* Received by the editors 7 April 2004. Accepted for publication 22 September 2004. Handling Editor: Harm Bart.

${ }^{\dagger}$ Fakultät II; Institut für Mathematik, Technische Universität Berlin, D-10623 Berlin, Germany (mehl@math.tu-berlin.de). A large part of this work was performed while this author was visiting the Vrije Universiteit Amsterdam and while he was supported by the Research Training Network HPRN-CT-2000-00116 of the European Union, "Classical Analysis, Operator Theory, Geometry of Banach Spaces, their interplay and their applications".

${ }^{\ddagger}$ Afdeling Wiskunde, Faculteit der Exacte Wetenschappen, Vrije Universiteit Amsterdam, De Boelelaan 1081a, 1081 HV Amsterdam, The Netherlands (ran@cs.vu.nl).

$\S$ College of William and Mary, Department of Mathematics, P. O. Box 8795, Williamsburg, VA 23187-8795, USA (lxrodm@math.wm.edu). The research of this author is partially supported by NSF Grant DMS-9988579. 
$\left[X^{[*]} x, y\right]$ for all $x, y \in \mathbb{C}^{n}$. We recall the following well studied classes of matrices that are structured with respect to the indefinite inner product, namely:

(a) $H$-selfadjoint matrices $X: X^{*} H=H X$;

(b) $H$-skew-adjoint matrices $X: X^{*} H=-H X$;

(c) $H$-unitary matrices $X: X^{*} H X=H$;

(d) $H$-dissipative matrices $X: \operatorname{Im}[X x, x]:=\left\langle\frac{1}{2 i}\left(H X-X^{*} H\right) x, x\right\rangle \geq 0$ for every $x \in \mathbb{C}^{n}$

(e) $H$-expansive matrices $X:[X x, X x] \geq[x, x]$ for every $x \in \mathbb{C}^{n}$.

(f) $H$-normal matrices $X: X^{[*]} X=X X^{[*]}$.

By analogy with the well-known class of hyponormal operators in Hilbert spaces, we introduce also the following class:

(g) $H$-hyponormal matrices $X: H\left(X^{[*]} X-X X^{[*]}\right) \geq 0$.

We note that in each case it is easy to check that if $A$ is from one of the classes of matrices in (a) $-(\mathrm{g})$ with respect to the inner product induced by $H$, then $P^{-1} A P$ is in the corresponding class with respect to the inner product induced by $P^{*} H P$, provided that $P$ is nonsingular.

It is well known that several classes of matrices $X$ in indefinite inner product spaces allow extensions of invariant $H$-nonnegative subspaces to invariant maximal $H$-nonnegative subspaces; in other words, if $\mathcal{M}_{0}$ is an $X$-invariant $H$-nonnegative subspace, then there exists an $X$-invariant maximal $H$-nonnegative subspace $\mathcal{M}$ that contains $\mathcal{M}_{0}$. Those classes are for example $H$-expansive matrices (including $\mathrm{H}$ unitary matrices) and $H$-dissipative matrices (including $H$-selfadjoint and $H$-skewadjoint matrices); see, e.g., [13] for a proof, as well as [1] and [7]. The natural question arises if this extension problem still has a solution for $H$-normal matrices. The answer is affirmative if the subspace $\mathcal{M}_{0}$ is also invariant for $X^{[*]}$; see Theorem 2.2 in Section 2. In the general case, a partial answer to this question has been given in [13].

Theorem 1.1. Let $H$ be invertible, let $X \in \mathbb{C}^{n \times n}$ be $H$-normal, and let $\mathcal{M}_{0}$ be an $H$-neutral $X$-invariant subspace. Then there exists an $X$-invariant maximal $H$-nonnegative subspace $\mathcal{M}$ such that $\mathcal{M}_{0} \subseteq \mathcal{M}$.

Theorem 1.1 follows immediately from a more general result (see [13, Theorem 6.3]). The proof of this result depends essentially on the H-neutrality of the given invariant subspace $\mathcal{M}_{0}$.

Theorem 1.1 also follows easily from a general result due to H. Langer [9], [10] concerning extension of dual pairs (we are indebted to $\mathrm{H}$. Langer for pointing out to us this observation and its proof). Indeed, let $\mathcal{M}_{0}$ be as in Theorem 1.1. Although $\mathcal{M}_{0}$ itself need not be $X^{[*]}$-invariant, the subspace

$$
\mathcal{M}_{1}:=\sum_{j=0}^{\infty}\left(X^{[*]}\right)^{j} \mathcal{M}_{0}
$$

is invariant for both $X$ and $X^{[*]}$. Moreover, $\mathcal{M}_{1}$ is $H$-neutral. To verify this, note that

$$
\left[\left(X^{[*]}\right)^{i} x,\left(X^{[*]}\right)^{j} y\right]=\left[X^{j} x, X^{i} y\right], \quad \forall x, y \in \mathbb{C}^{n \times n}, \quad i, j=0,1, \ldots
$$


in view of the $H$-normality of $X$. Therefore for $x_{1}, \ldots, x_{p}, y_{1}, \ldots, y_{q} \in \mathcal{M}_{0}$ we have

$$
\left[\sum_{j=0}^{p} \alpha_{j}\left(X^{[*]}\right)^{j} x_{j}, \sum_{k=0}^{q} \beta_{k}\left(X^{[*]}\right)^{k} y_{k}\right]=\sum_{j, k=0}^{j=p, k=q} \alpha_{j} \overline{\beta_{k}}\left[X^{k} x_{j}, X^{j} y_{k}\right]=0
$$

since $\mathcal{M}_{0}$ is $X$-invariant and $H$-neutral. Now by [9], [10], $\mathcal{M}_{1}$ can be extended to a maximal $H$-nonnegative subspace that is invariant for both $X$ and $X^{[*]}$. This proof shows that Theorem 1.1 is valid also for normal operators in Pontryagin spaces; see $[11]$.

After discussing some preliminary results on invariant maximal semidefinite subspaces for $H$-normal matrices in Section 2, we give an example in Section 3 showing that Theorem 1.1 does not hold true if we replace the $H$-neutral $X$-invariant subspace $\mathcal{M}_{0}$ by an $H$-nonnegative $X$-invariant subspace. On the other hand, we show in Section 4 that the extension problem has a positive solution if we start with an invariant $H$-definite subspace and if additional hypotheses are satisfied. We prove the extension results in the context of $H$-hyponormal matrices. Throughout the paper, let $e_{1}, \ldots, e_{n}$ be the canonical unit vectors of $\mathbb{C}^{n}: e_{j}$ has 1 in the $j$ th position and zeros elsewhere.

2. Normal matrices and invariant maximal semidefinite subspaces. In this section, we present some results on invariant maximal semidefinite subspaces of $H$-normal matrices, starting with the following.

Proposition 2.1. Let $X \in \mathbb{C}^{n \times n}$ be $H$-normal and let $\mathcal{M}$ be an $X$-invariant maximal $H$-nonnegative subspace. Then $\mathcal{M}$ is invariant also for $X^{[*]}$.

Proof. Applying otherwise a suitable transformation $X \mapsto P^{-1} X P, H \mapsto$ $P^{*} H P$, where $P$ is invertible, we may assume that $\mathcal{M}$ is spanned by the first (say) $m$ unit vectors and that $X$ and $H$ have the forms

$$
X=\left[\begin{array}{cccc}
X_{11} & X_{12} & X_{13} & X_{14} \\
X_{21} & X_{22} & X_{23} & X_{24} \\
0 & 0 & X_{33} & X_{34} \\
0 & 0 & X_{43} & X_{44}
\end{array}\right], \quad H=\left[\begin{array}{cccc}
I & 0 & 0 & 0 \\
0 & 0 & I & 0 \\
0 & I & 0 & 0 \\
0 & 0 & 0 & -I
\end{array}\right]
$$

Indeed, this follows easily by decomposing $\mathcal{M}=\mathcal{M}_{p} \oplus \mathcal{M}_{0}$ into an $H$-neutral subspace $\mathcal{M}_{0}$ and its orthogonal complement $\mathcal{M}_{p}$ (in $\left.\mathcal{M}\right)$, and choosing an $H$-neutral subspace $\mathcal{M}_{s l}$ that is skewly linked to $\mathcal{M}_{0}$ (see [8], [12]). Note that the $H$-orthogonal complement to $\mathcal{M} \dot{+} \mathcal{M}_{s l}$ is necessarily an $H$-negative subspace due to the maximality of $\mathcal{M}$. Then, selecting appropriate bases in all subspaces constructed above, and putting the bases as the consecutive columns of a matrix $P$, we get a transformation that yields the desired result. (Decompositions analogous to (2.1) have been used in the literature; see, e.g., [13].) From (2.1), we then obtain that

$$
X^{[*]}=\left[\begin{array}{cccc}
X_{11}^{*} & 0 & X_{21}^{*} & 0 \\
X_{13}^{*} & X_{33}^{*} & X_{23}^{*} & -X_{43}^{*} \\
X_{12}^{*} & 0 & X_{22}^{*} & 0 \\
-X_{14}^{*} & -X_{34}^{*} & -X_{24}^{*} & X_{44}^{*}
\end{array}\right]
$$


and

$$
\begin{aligned}
& X^{[*]} X-X X^{[*]}= \\
& {\left[\begin{array}{cccc}
* & * & * & * \\
* & * & * & * \\
* & X_{12}^{*} X_{12}+X_{34} X_{34}^{*} & * & * \\
* & * & * & X_{44}^{*} X_{44}-X_{14}^{*} X_{14}-X_{24}^{*} X_{34}-X_{34}^{*} X_{24}-X_{44} X_{44}^{*}
\end{array}\right]}
\end{aligned}
$$

Since $X$ is $H$-normal, i.e., $X^{[*]} X-X X^{[*]}=0$, we obtain from the block $(3,2)$-entry in (2.3) that $X_{12}=0$ and $X_{34}=0$. But then the equation for the block $(4,4)$-entry of (2.3) becomes

$$
X_{44}^{*} X_{44}-X_{44} X_{44}^{*}=X_{14}^{*} X_{14} \geq 0
$$

which is easily seen to imply (by taking traces of both sides in (2.4)) that $X_{14}=0$. Thus, we obtain from (2.2) that $\mathcal{M}$ is also invariant for $X^{[*]}$.

The following result is well known; see [2], for example.

TheOREM 2.2. Let $X$ be $H$-normal and let $\mathcal{M}_{0}$ be an $X$-invariant $H$-nonnegative subspace that is also invariant for $X^{[*]}$. Then there exists an $X$-invariant maximal $H$-nonnegative subspace $\mathcal{M}$ containing $\mathcal{M}_{0}$.

In general, an $X$-invariant $H$-nonnegative subspace need not be invariant for $X^{[*]}$. However, for a particular subclass of the set of $H$-normal matrices this is always the case. An $H$-normal matrix is called polynomially $H$-normal if $X^{[*]}$ is a polynomial in $X$. See [14] for information on this and related classes of $H$-normal matrices. Clearly, if $X$ is polynomially $H$-normal then any subspace that is invariant for $X$ is also invariant for $X^{[*]}$. Thus, we immediately obtain the following corollary to Theorem 2.2:

TheOREM 2.3. Let $X$ be polynomially $H$-normal and let $\mathcal{M}_{0}$ be an $X$-invariant $H$-nonnegative subspace. Then there exists an $X$-invariant maximal $H$-nonnegative subspace $\mathcal{M}$ containing $\mathcal{M}_{0}$.

In the next section, we construct an example showing that Theorem 2.3 does not hold for $H$-normal matrices in general.

3. An example. Let $b, h \in \mathbb{R}$ and set

$$
X=\left[\begin{array}{cccc}
0 & b & -b h & -\frac{h^{2}}{2} \\
1 & h & -\frac{h^{2}}{2} & h \\
0 & 0 & 0 & 1 \\
0 & 0 & b & h
\end{array}\right], \quad H=\left[\begin{array}{llll}
0 & 0 & 1 & 0 \\
0 & 1 & 0 & 0 \\
1 & 0 & 0 & 0 \\
0 & 0 & 0 & 1
\end{array}\right]
$$

Then it is easy to check that $X$ is $H$-normal. Moreover, $\mathcal{M}_{0}:=\operatorname{Span}\left(e_{1}, e_{2}\right)$ is an $H$-nonnegative subspace and is invariant for $X$. However, there does not exist an $X$-invariant maximal $H$-nonnegative subspace that contains $\mathcal{M}_{0}$, because the only $H$ nonnegative subspace of dimension three that contains $\mathcal{M}_{0}$ is $\mathcal{M}:=\operatorname{Span}\left(e_{1}, e_{2}, e_{4}\right)$. This subspace, however, is not $X$-invariant. 
It turns out that the matrix $X$ provides additional counterexamples, as well as illustrates Theorem 1.1. For these reasons, we will study in detail the structure of $X$-invariant $H$-nonnegative subspaces.

1. Spectral information on $X$ : The characteristic polynomial of $X$ is $\left(\lambda^{2}-\lambda h-b\right)^{2}$, so that there are (generically) two eigenvalues, each with geometric multiplicity two. We shall denote these by $\lambda_{1}$ and $\lambda_{2}$, and if they are real, we shall always assume that $\lambda_{1} \leq \lambda_{2}$. To avoid the non-generic case we shall assume in the sequel that $h^{2}+4 b \neq 0$ and $b \neq 0$. In that case zero is not an eigenvalue, and there are precisely two eigenvalues, which are given by given by

$$
\lambda_{1,2}=\frac{h}{2} \pm \sqrt{\frac{h^{2}+4 b}{4}} .
$$

Moreover, $X$ is diagonalizable. A basis of eigenvectors corresponding to $\lambda_{i}$ is given by the two vectors

$$
\begin{aligned}
x_{i}: & =\left[\begin{array}{llll}
\lambda_{i}-h & 1 & 0 & 0
\end{array}\right]^{T}=\left[\begin{array}{llll}
-\lambda_{3-i} & 1 & 0 & 0
\end{array}\right]^{T}, \\
y_{i}: & =\left[\begin{array}{llll}
-\frac{b h}{\lambda_{i}}-\frac{h^{2}}{2} & 0 & 1 & \lambda_{i}
\end{array}\right]^{T}=\left[\begin{array}{llll}
\frac{1}{2}\left(\lambda_{3-i}^{2}-\lambda_{i}^{2}\right) & 0 & 1 & \lambda_{i}
\end{array}\right]^{T} .
\end{aligned}
$$

(Here, the superscript ${ }^{T}$ denotes the transposed vector, and we use the fact that $h=\lambda_{i}+\lambda_{3-i}$ and that $b=-\lambda_{i} \lambda_{3-i}$.) We shall denote the spectral subspace spanned by the eigenvectors corresponding to $\lambda_{i}$ by $\mathcal{M}_{i}$.

It is now easy to see that any three-dimensional $X$-invariant subspace $\mathcal{M}$ is of the following form: $\mathcal{M}$ is the span of $\mathcal{M}_{i}$ (for either $i=1$ or $i=2$ ) and of one other vector $z$ of the form $z=[\alpha, 0,0, \delta]^{T}(\alpha, \delta \in \mathbb{C}$, not both zeros). The reasoning behind this is that we can write $z$ as a linear combination of vectors in $\mathcal{M}_{i}$ and one fixed vector $\tilde{x}_{i}$ in $\mathcal{M}_{3-i}$. As the latter is an eigenvector, we find that $X z$ is in the span of $\mathcal{M}_{i}$ and $\tilde{x}_{i}$, that is, in the span of $\mathcal{M}_{i}$ and $z$. By scaling $z$ we may assume that either $z=z_{\alpha}=[\alpha, 0,0,1]^{T}$, or $z=e_{1}$. In the former case we shall denote the span of $\mathcal{M}_{i}$ and $z_{\alpha}$ by $\mathcal{M}_{i, \alpha}$, in the latter case we denote the span of $e_{1}$ and $\mathcal{M}_{i}$ by $\mathcal{M}_{i, e}$.

2. Invariant maximal $H$-nonnegative subspaces: We now consider the question under which conditions the spaces $\mathcal{M}_{i, \alpha}$ and $\mathcal{M}_{i, e}$ are $H$-nonnegative or $H$-positive. We do this by computing the Gram matrix of the $H$-inner product on this space with respect to the basis $x_{i}, y_{i}, z_{\alpha}$, respectively, $x_{i}, y_{i}, e_{1}$. We start with $\mathcal{M}_{i, e}$. Since $\left\langle H y_{i}, e_{1}\right\rangle=1$ and $\left\langle H e_{1}, e_{1}\right\rangle=0$ the Gram matrix is indefinite. So $\mathcal{M}_{i, e}$ is $H$-indefinite for both $i=1$ and $i=2$.

Next, consider $\mathcal{M}_{i, \alpha}$. One easily computes that

$$
\begin{gathered}
\left\langle H x_{i}, x_{i}\right\rangle=1, \quad\left\langle H x_{i}, y_{i}\right\rangle=-\lambda_{3-i}, \\
\left\langle H x_{i}, z_{\alpha}\right\rangle=0, \quad\left\langle H y_{i}, z_{\alpha}\right\rangle=\lambda_{i}+\bar{\alpha}, \quad\left\langle H z_{\alpha}, z_{\alpha}\right\rangle=1,
\end{gathered}
$$

and

$$
\left\langle H y_{i}, y_{i}\right\rangle=\operatorname{Re}\left(\lambda_{3-i}^{2}-\lambda_{i}^{2}\right)+\left|\lambda_{i}^{2}\right|=: \beta_{i}
$$


Hence, the Gram matrix of $H$ on $\mathcal{M}_{i, \alpha}$ is given by:

$$
G_{i \alpha}=\left[\begin{array}{ccc}
1 & -\bar{\lambda}_{3-i} & 0 \\
-\lambda_{3-i} & \beta_{i} & \bar{\lambda}_{i}+\alpha \\
0 & \lambda_{i}+\bar{\alpha} & 1
\end{array}\right] .
$$

The subspace $\mathcal{M}_{i, \alpha}$ is $H$-nonnegative if and only if $G_{i \alpha} \geq 0$. Clearly, this is the case if and only if the determinants of all principal minors are nonnegative. This amounts to the following condition:

$$
\beta_{i}-\left|\lambda_{i}+\bar{\alpha}\right|^{2}-\left|\lambda_{3-i}\right|^{2} \geq 0 .
$$

We consider two cases separately. First we assume that the eigenvalues $\lambda_{i}$ are nonreal. In that case $b$ must be negative, and $\lambda_{1,2}=\frac{h}{2} \pm i \sqrt{\frac{-4 b-h^{2}}{4}}$, and so $\operatorname{Re}\left(\lambda_{3-i}^{2}-\right.$ $\left.\lambda_{i}^{2}\right)=0$. It follows that $\beta_{i}=\left|\lambda_{i}\right|^{2}=\left|\lambda_{3-i}\right|^{2}$. In that case there is only one choice of $\alpha$ for which $G_{i \alpha} \geq 0$, namely, $\alpha=-\bar{\lambda}_{i}$.

Next, we assume that $\lambda_{i}$ is real, that is $h^{2}+4 b>0$. In that case $\beta_{i}=\left(\lambda_{3-i}^{2}-\right.$ $\left.\lambda_{i}^{2}\right)+\lambda_{i}^{2}=\lambda_{3-i}^{2}$. Hence, $G_{i \alpha} \geq 0$ if and only if $0 \geq\left|\lambda_{i}+\bar{\alpha}\right|^{2}=\left|\alpha+\lambda_{i}\right|^{2}$. Again, there is only one choice of $\alpha$ that will make $G_{i \alpha} \geq 0$, namely, $\alpha=-\lambda_{i}$.

Note that in particular, the preceding arguments show that the two spectral subspaces $\mathcal{M}_{i}$ are $H$-nonnegative.

Concluding, we see that all the maximal $H$-nonnegative $X$-invariant subspaces are of the form $\mathcal{M}_{i, \alpha}$ with $i=1,2$ and $\alpha=-\bar{\lambda}_{i}$. In particular, in the generic case when $\lambda_{1} \neq \lambda_{2}$ there are just two $X$-invariant maximal $H$-nonnegative subspaces.

3. Not every $H$-positive $X$-invariant subspace $\mathcal{M}_{p}$ can be extended to a maximal $H$-nonnegative $X$-invariant subspace: For this we take $h>0$ and $b>0$. Then the two eigenvalues $\lambda_{1}<\lambda_{2}$ are both real. Consider the space $\mathcal{M}_{p}=\operatorname{Span}\left(x_{2}, y_{1}\right)$. The Gram matrix of $H$ with respect to this basis is

$$
\left[\begin{array}{cc}
1 & -\lambda_{1} \\
-\lambda_{1} & \lambda_{2}^{2}
\end{array}\right]
$$

This is positive definite, since the determinant is equal to $\lambda_{2}^{2}-\lambda_{1}^{2}$. So, $\mathcal{M}_{p}$ is $H$ positive. Note that an $X$-invariant maximal $H$-nonnegative subspace containing $\mathcal{M}_{p}$ must necessarily be of the form $\mathcal{M}_{1,-\lambda_{1}}$ or $\mathcal{M}_{2,-\lambda_{2}}$. So, assume first that $\mathcal{M}_{p}$ is contained in $\mathcal{M}_{1,-\lambda_{1}}$. Then $x_{2} \in \mathcal{M}_{1,-\lambda_{1}}$. That is, $x_{2}=c_{1} x_{1}+c_{2} y_{1}+c_{3} z_{-\lambda_{1}}$ for some $c_{1}, c_{2}$, and $c_{3}$. Writing this out in coordinates, one immediately sees that $c_{1}=1, c_{2}=0$, and $c_{3}=0$, which leads to a contradiction since $\lambda_{1} \neq \lambda_{2}$. So, assume that $\mathcal{M}_{p}$ is contained in $\mathcal{M}_{2,-\lambda_{2}}$. Then $y_{1} \in \mathcal{M}_{2,-\lambda_{2}}$. Hence $y_{1}=c_{1} x_{2}+c_{2} y_{2}+c_{3} z_{-\lambda_{2}}$ for some $c_{1}, c_{2}$, and $c_{3}$. Writing this out in coordinates, one immediately sees that $c_{1}=0, c_{2}=1$, and $c_{3}=\lambda_{1}-\lambda_{2}$, from the second, third, and fourth coordinates, respectively. The equation for the first coordinate then becomes

$$
\frac{1}{2}\left(\lambda_{2}^{2}-\lambda_{1}^{2}\right)=\frac{1}{2}\left(\lambda_{1}^{2}-\lambda_{2}^{2}\right)-\lambda_{2}\left(\lambda_{1}-\lambda_{2}\right) .
$$


One easily sees that this is equivalent to $\lambda_{1}=0$. However, since $b>0$, this cannot be the case. Again we arrive at a contradiction, and so we conclude that the $X$-invariant $H$-positive subspace $\mathcal{M}_{p}$ is not contained in any $X$-invariant maximal $H$-nonnegative subspace.

4. Every $X$-invariant $H$-neutral subspace admits an extension to an $X$-invariant maximal $H$-nonnegative subspace (as it should by Theorem 1.1): We only verify this for the generic case $h^{2}+4 b \neq 0$ and $b \neq 0$. Since $H$ has three positive and only one negative eigenvalue, the maximal dimension for an $H$-neutral subspace is one. Hence, every $X$-invariant $H$-neutral subspace $\mathcal{N}_{0}$ is contained in either $\mathcal{M}_{1}$ or $\mathcal{M}_{2}$. Both these subspaces are $H$-nonnegative. They are contained in $\mathcal{M}_{1,-\bar{\lambda}_{1}}$, respectively, $\mathcal{M}_{2,-\bar{\lambda}_{2}}$. So, $\mathcal{N}_{0}$ is contained in either $\mathcal{M}_{1,-\bar{\lambda}_{1}}$ or in $\mathcal{M}_{2,-\bar{\lambda}_{2}}$, and these spaces are $X$-invariant and maximal $H$-nonnegative.

5. In the generic case the matrix $X$ is block Toeplitz but not polynomially $H$-normal: Recall that an $H$-normal matrix $Y$ is said to be block Toeplitz if the indecomposable components $Y_{1}, \ldots, Y_{k}$ of $Y$ have the property that the Jordan form of each $Y_{i}$ consists either of exactly one Jordan block, or of exactly two Jordan blocks, and in the latter case the two Jordan blocks correspond to different eigenvalues; see [5], [6]. It was shown in [14] that the class of block Toeplitz $H$-normal matrices strictly contains the class of polynomially $H$-normal matrices. A computation now shows that

$$
X^{[*]}=\left[\begin{array}{cccc}
0 & -\frac{h^{2}}{2} & -b h & b \\
0 & h & b & 0 \\
0 & 1 & 0 & 0 \\
1 & h & -\frac{h^{2}}{2} & h
\end{array}\right]
$$

and therefore, $X$ is clearly not polynomially $H$-normal. Next, the description (given in [4]) of all indecomposable $H$-normal matrices in the case when $H$ has only one negative eigenvalue shows that in this case all diagonalizable $H$-normal matrices are block Toeplitz.

Concluding this section, we note that the example shows that Theorem 2.3 does not hold true for $H$-normal matrices in general, not even when specialized to $H$ positive invariant subspaces, nor when restricted to the smaller class of block Toeplitz $H$-normal matrices.

4. $H$-hyponormal matrices. In this section, we investigate under which conditions invariant $H$-positive subspaces of $H$-normal matrices can be extended to invariant maximal $H$-nonnegative subspaces. We do this in the more general context of $H$-hyponormal matrices. We start with a characterization of $H$-hyponormal matrices.

Proposition 4.1. Let $X \in \mathbb{C}^{n \times n}$ and let $A=\frac{1}{2}\left(X+X^{[*]}\right)$ and $S=\frac{1}{2}(X-$ $\left.X^{[*]}\right)$ denote its $H$-selfadjoint and $H$-skew-adjoint parts, respectively. Then $X$ is $H$ hyponormal if and only if $B:=i S$ is $H A$-dissipative.

Proof. Note that $X=A+S=A-i B$ and $X^{[*]}=A-S=A+i B$. Then

$$
X^{[*]} X-X X^{[*]}=(A+i B)(A-i B)-(A-i B)(A+i B)=2 i(B A-A B)
$$


Since $B$ is $H$-selfadjoint, we obtain that

$$
H\left(X^{[*]} X-X X^{[*]}\right)=2 i\left(B^{*} H A-H A B\right) .
$$

Hence $X$ is $H$-hyponormal if and only if $B$ is $H A$-dissipative.

Note that $H A$ in Proposition 4.1 is Hermitian, but not necessarily invertible. In this situation, the definition of dissipative matrices given in the introduction applies as well. We mention in passing that simple forms for $H$-dissipative matrices were obtained in [15].

Theorem 4.2. Let $X$ be $H$-hyponormal and let $A=\frac{1}{2}\left(X+X^{[*]}\right)$ and $S=$ $\frac{1}{2}\left(X-X^{[*]}\right)$ denote its $H$-selfadjoint and $H$-skew-adjoint parts, respectively.

1. If the spectral subspace of $A$ associated with the real spectrum of $A$ is not $H$ negative (not $H$-positive, respectively), then there exists a common eigenvector of $A$ and $S$ that corresponds to a real eigenvalue of $A$ and is $H$-nonnegative (H-nonpositive, respectively).

2. If the spectral subspace of $S$ associated with the purely imaginary (possibly including zero) spectrum of $S$ is not $H$-negative (not $H$-positive, respectively), then there exists a common eigenvector of $A$ and $S$ that corresponds to a purely imaginary eigenvalue of $S$ and is $H$-nonnegative ( $H$-nonpositive, respectively).

Proof. The following notation will be used in the proof: $\mathcal{J}_{m}(\mu)$ is the $m \times m$ upper triangular Jordan block with the eigenvalue $\mu$ and $Z_{m}$ is the $m \times m$ matrix having ones on the lower left - upper right anti-diagonal and zeros everywhere else.

We only prove 1 ). The proof for 2 ) then follows by considering $i X$. Applying a suitable transformation otherwise, we may assume that $(A, H)$ is in a canonical form (the canonical form is well-known; see, e.g., [3]). Without loss of generality, we may assume that the spectral subspace of $A$ associated with the real spectrum of $A$ is not $H$-negative. (Otherwise, replace $H$ with $-H$, and consider $X^{[*]}$ in place of $X$.) Then there is at least one $H$-nonnegative eigenvector $v$ for $A$ associated with a real eigenvalue $\lambda$ of $A$. (Indeed, if all eigenvectors of $A$ associated with real eigenvalues would be $H$-negative, then it follows from the canonical form for $(A, H)$ that $A$ has no Jordan blocks of size larger than one associated with real eigenvalues. But then, the spectral subspace with respect to the real eigenvalues of $A$ would be $H$-negative. Recall also that by default the zero subspace is $H$-negative, so the assumption of the theorem implies in particular that $A$ does have real eigenvalues.) Assume without loss of generality that $\lambda=0$ (otherwise subtract $\lambda I$ from $X$ ), and furthermore that

$$
A=A_{1} \oplus \cdots \oplus A_{k}, \quad H=H_{1} \oplus \cdots \oplus H_{k},
$$

where $A_{1}=\mathcal{J}_{n}(0)$, and where $H_{1}=\varepsilon Z_{n}, \varepsilon= \pm 1$. Let

$$
i S=B=\left[\begin{array}{ccc}
B_{11} & \cdots & B_{1 k} \\
\vdots & \ddots & \vdots \\
B_{k 1} & \cdots & B_{k k}
\end{array}\right]
$$


be partitioned conformably with $A$ and $H$. Since $B$ is $H$-selfadjoint, we have, in particular, that $B_{1 j}^{*} H_{1}=H_{j} B_{j 1}$. Furthermore, Proposition 4.1 implies that

$$
\begin{aligned}
C & :=i\left(B^{*} H A-H A B\right) \\
& =i\left[\begin{array}{ccc}
B_{11}^{*} H_{1} A_{1}-H_{1} A_{1} B_{11} & \cdots & B_{k 1}^{*} H_{k} A_{k}-H_{1} A_{1} B_{1 k} \\
\vdots & \ddots & \vdots \\
B_{1 k}^{*} H_{1} A_{1}-H_{k} A_{k} B_{k 1} & \cdots & B_{k k}^{*} H_{k} A_{k}-H_{k} A_{k} B_{k k}
\end{array}\right] \geq 0 .
\end{aligned}
$$

In particular, $C_{11}:=i\left(B_{11}^{*} H_{1} A_{1}-H_{1} A_{1} B_{11}\right) \geq 0$. Let

$$
B_{11}=\left[\begin{array}{ccc}
b_{11} & \cdots & b_{1 n} \\
\vdots & \ddots & \vdots \\
b_{n 1} & \cdots & b_{n n}
\end{array}\right]
$$

Then the fact that $B$ is $H$-selfadjoint implies that $B_{11}$ is $H_{1}$-selfadjoint, i.e., $b_{n 1}$ is real and $\bar{b}_{n j}=b_{n-j+1,1}$ for $j=2, \ldots, n$. Moreover, the $(1,1)$-entry of $C_{11}$ (and of $C$ ) is zero. Hence, the first column of $C_{11}$ (and also of $C$ ) is necessarily zero. Computing the $(j, 1)$-entry of $C_{11}$ for $j>1$, we then obtain that $0=-\varepsilon b_{n-j+2,1}$. Hence $b_{j 1}=0$ for $j=2, \ldots, n$. Next, we will investigate the structure of the blocks $B_{l 1}, l>1$, by using the information that the first column of

$$
C_{l 1}:=i\left(B_{1 l}^{*} H_{1} A_{1}-H_{l} A_{l} B_{l 1}\right)=i H_{l}\left(B_{l 1} A_{1}-A_{l} B_{l 1}\right)
$$

and hence also of the matrix $D:=B_{l 1} A_{1}-A_{l} B_{l 1}$ is necessarily zero. Let $B_{l 1}=$ $\left(\tilde{b}_{r s}\right) \in \mathbb{C}^{m \times n}$. We will then distinguish two different cases.

Case (1): $A_{l}=\mathcal{J}_{m}(\mu), H_{l}=\delta Z_{m}$, where $\mu$ is real and $\delta= \pm 1$. Then the $(j, 1)$-entry $d_{j 1}$ of $D$ has the form

$$
0=d_{j 1}= \begin{cases}-\mu \tilde{b}_{j 1}-\tilde{b}_{j+1,1} & \text { for } j<m \\ -\mu \tilde{b}_{m 1} & \text { for } j=m .\end{cases}
$$

Thus, if $\mu \neq 0$, then the first column of $B_{l 1}$ is zero, and if $\mu=0$, then the first column of $B_{l 1}$ is zero except for maybe $\tilde{b}_{11}$.

Case (2): $A_{l}=\mathcal{J}_{p}(\mu) \oplus \mathcal{J}_{p}(\bar{\mu})$ and $H_{l}=Z_{2 p}$, where $\mu$ is nonreal and $m=2 p$ is even. Then the $(j, 1)$-entry $d_{j 1}$ of $D$ has the form

$$
0=d_{j 1}= \begin{cases}-\mu \tilde{b}_{j 1}-\tilde{b}_{j+1,1} & \text { for } j<p \\ -\mu \tilde{b}_{p 1} & \text { for } j=p \\ -\bar{\mu} \tilde{b}_{j 1}-\tilde{b}_{j+1,1} & \text { for } p<j<m \\ -\bar{\mu} \tilde{b}_{m 1} & \text { for } j=m .\end{cases}
$$

Since $\mu, \bar{\mu} \neq 0$, we obtain that the first column of $B_{l 1}$ is zero.

Note that the argument above can also be applied to all other Jordan blocks of $A$ that are associated with the eigenvalue zero and the first columns to the corresponding blocks in the matrix $B$. Thus, if $A$ has $p$ Jordan blocks associated with the eigenvalue 
0 and if we apply a permutation that groups together all first columns of these Jordan blocks, we finally obtain that $A, S=-i B$, and $H$ have the forms

$$
A=\left[\begin{array}{ll}
0 & A_{12} \\
0 & A_{22}
\end{array}\right], \quad S=\left[\begin{array}{cc}
S_{11} & S_{12} \\
0 & S_{22}
\end{array}\right], \quad H=\left[\begin{array}{cc}
H_{11} & H_{12} \\
H_{21} & H_{22}
\end{array}\right]
$$

where $S_{11} \in \mathbb{C}^{p \times p}$, and $A_{22} \in \mathbb{C}^{(n-p) \times(n-p)}$, i.e., the eigenspace of $A$ associated with zero is also invariant for $S$. By the choice of the eigenvalue zero this eigenspace is not $H$-negative, i.e., $H_{11}$ is not negative definite. Note that $S_{11}$ is $H_{11}$-skewadjoint. Suppose that all eigenvectors of $-i S_{11}$ are $H_{11}$-negative. By considering the canonical form of the pair $\left(-i S_{11}, H_{11}\right)$ (see, e.g, [3]) we see that this implies that $H_{11}$ is negative definite. As this is not the case, $S_{11}$ must have an eigenvector that is $H_{11}$-nonnegative. Extending this eigenvector to the full space in the canonical way, we obtain an $H$-nonnegative eigenvector for $S$ that is obviously also an eigenvector for $A$. $\quad$ प

TheOREM 4.3. Let $X \in \mathbb{C}^{n \times n}$ be $H$-hyponormal and let $A=\frac{1}{2}\left(X+X^{[*]}\right)$ and $S=\frac{1}{2}\left(X-X^{[*]}\right)$ denote the $H$-selfadjoint and $H$-skew-adjoint parts of $X$, respectively. If the spectrum of $A$ is real or if the spectrum of $S$ is purely imaginary (possibly including zero), then there exist an $X$-invariant maximal $H$-nonnegative subspace and an $X$-invariant maximal $H$-nonpositive subspace that are both also invariant for $X^{[*]}$ (and for $A$ and $S$ ).

Proof. We only prove the existence of an $X$-invariant maximal $H$-nonnegative subspace that is also invariant for $X^{[*]}$. The corresponding result for a maximal $H$ nonpositive subspace follows analogously, by replacing $H$ and $X$ with $-H$ and $X^{[*]}$, respectively. Without loss of generality we may assume that the spectrum of $A$ is real. (Otherwise consider $i X$.) The proof then proceeds via induction on $n$. For $n=1$, there is nothing to show. Hence, assume $n>1$. Let $A=\frac{1}{2}\left(X+X^{[*]}\right)$ and $S=\frac{1}{2}\left(X-X^{[*]}\right)$ denote the $H$-selfadjoint and $H$-skew-adjoint parts of $X$, respectively. If $H$ is negative definite, then there is nothing to show. Otherwise, Theorem 4.2 shows that there exists an $H$-nonnegative vector $v$ that is a common eigenvector of $A$ and $S$. We now distinguish two cases.

Case (1): $v$ is $H$-positive. Applying a suitable transformation otherwise, we may assume that $v=e_{1}$ and that $A, S$, and $H$ take the forms

$$
A=\left[\begin{array}{cc}
a_{11} & A_{12} \\
0 & A_{22}
\end{array}\right], \quad S=\left[\begin{array}{cc}
s_{11} & S_{12} \\
0 & S_{22}
\end{array}\right], \quad H=\left[\begin{array}{cc}
1 & 0 \\
0 & H_{22}
\end{array}\right],
$$

where $a_{11}, s_{11} \in \mathbb{C}$. Then the fact that $A$ is $H$-selfadjoint and $S$ is $H$-skew-adjoint implies that $A_{12}=0=S_{12}$. One easily checks that $X_{22}$ is $H_{22}$-hyponormal, that $A_{22}$ is its $H_{22}$-selfadjoint part, and that $S_{22}$ is its $H_{22}$-skew-adjoint part. Moreover, the spectrum of $A_{22}$ is real. By the induction hypothesis, there exists a maximal $H_{22}$-nonnegative subspace $\mathcal{M}_{0}$ that is invariant for both $A_{22}$ and $S_{22}$. Then clearly $\mathcal{M}=\operatorname{Span}\left(e_{1}, \mathcal{M}_{0}\right)$ is maximal $H$-nonnegative and invariant for $X^{[*]}$ and both $A$ and $S$.

Case (2): $v$ is $H$-neutral. Again, we may assume that $v=e_{1}$ and, moreover, that 
$A, S$, and $H$ take the forms

$$
A=\left[\begin{array}{ccc}
a_{11} & a_{12} & A_{13} \\
0 & a_{22} & A_{23} \\
0 & A_{32} & A_{33}
\end{array}\right], \quad S=\left[\begin{array}{ccc}
s_{11} & s_{12} & S_{13} \\
0 & s_{22} & S_{23} \\
0 & S_{32} & S_{33}
\end{array}\right], \quad H=\left[\begin{array}{ccc}
0 & 1 & 0 \\
1 & 0 & 0 \\
0 & 0 & H_{33}
\end{array}\right],
$$

where $a_{11}, s_{11}, a_{22}, s_{22} \in \mathbb{C}$. Then the fact that $A$ is $H$-selfadjoint and that $S$ is $H$ skew-adjoint implies that $A_{23}=0=S_{23}$. It is easily seen that this implies that $X_{33}$ is $H_{33}$-hyponormal, and that its $H_{33}$-selfadjoint part is $A_{33}$, while its $H_{33}$-skew-adjoint part is $S_{33}$. Moreover, $\sigma(A)=\left\{a_{11}, a_{22}\right\} \cup \sigma\left(A_{33}\right)$, and $\sigma(S)=\left\{s_{11}, s_{22}\right\} \cup \sigma\left(S_{33}\right)$. Hence the spectrum of $A_{33}$ is real. So we can apply the induction hypothesis, by which there exists a maximal $H_{33}$-nonnegative subspace $\mathcal{M}_{0}$ that is invariant for both $A_{33}$ and $S_{33}$. Then clearly $\mathcal{M}=\operatorname{Span}\left(e_{1}, \mathcal{M}_{0}\right)$ is maximal $H$-nonnegative and invariant for $X^{[*]}$ and both $A$ and $S$.

The assumption that the spectral subspace $X+X^{[*]}$ is not $H$-negative (or not $H$-positive) in Theorem 4.2 and that the spectrum of $X+X^{[*]}$ is real in Theorem 4.3 is essential. The following example illustrates this.

EXAMPLE 1. Let

$$
H=\left[\begin{array}{ll}
0 & 1 \\
1 & 0
\end{array}\right], \quad A=\left[\begin{array}{cc}
i & 0 \\
0 & -i
\end{array}\right], \quad B=\left[\begin{array}{cc}
0 & 1 \\
-1 & 0
\end{array}\right]
$$

Then a straightforward calculation reveals that $A$ and $B$ are $H$-selfadjoint and that $B^{*} H A-H A B=-2 i I_{2}$, i.e., $B$ is $H A$-dissipative. Hence, $X=A-i B$ is $H$ hyponormal. On the other hand, there exists no common eigenvector of $A$ and $S=-i B$ and thus, also no maximal $H$-nonnegative subspace that is invariant for both $A$ and $S$.

Theorem 4.4. Let $X \in \mathbb{C}^{n \times n}$, and let $\mathcal{M}_{0}$ be an $X$-invariant subspace. Assume that at least one of the following two hypotheses holds true:

(A) $X$ is $H$-hyponormal and $\mathcal{M}_{0}$ is $H$-negative;

(B) $X$ is $H$-normal and $\mathcal{M}_{0}$ is $H$-positive.

Define

$$
X_{22}:=\left.P X\right|_{\mathcal{M}_{0}^{[\perp]}}: \mathcal{M}_{0}^{[\perp]} \rightarrow \mathcal{M}_{0}^{[\perp]}
$$

where

$$
\mathcal{M}_{0}^{[\perp]}:=\left\{x \in \mathbb{C}^{n \times n} \mid[x, y]=0 \quad \text { for } \text { every } y \in \mathcal{M}_{0}\right\},
$$

and where $P$ is the projection onto $\mathcal{M}_{0}^{[\perp]}$ along $\mathcal{M}_{0}$. Equip $\mathcal{M}_{0}^{[\perp]}$ with the indefinite inner product induced by $H$. Assume that

$$
\sigma\left(X_{22}+X_{22}^{[*]}\right) \subset \mathbb{R} \quad \text { or } \quad \sigma\left(X_{22}-X_{22}^{[*]}\right) \subset i \mathbb{R}
$$


Then there exists an $X$-invariant maximal $H$-nonpositive subspace ( if $(A)$ holds true), or an $X$-invariant maximal $H$-nonnegative subspace ( if $(B)$ holds true), that contains $\mathcal{M}_{0}$.

Proof. Without loss of generality we may assume that $\sigma\left(X_{22}^{[*]}+X_{22}\right) \subset \mathbb{R}$. We may moreover assume without loss of generality that $\mathcal{M}_{0}=\operatorname{Span}\left(e_{1}, \ldots, e_{k}\right)$ and that $X$ and $H$ have the following block forms:

$$
X=\left[\begin{array}{cc}
X_{11} & X_{12} \\
0 & X_{22}
\end{array}\right], \quad H=\left[\begin{array}{cc}
\varepsilon I_{k} & 0 \\
0 & H_{2}
\end{array}\right]
$$

where $\varepsilon=+1$ if $(B)$ holds and hence $\mathcal{M}_{0}$ is $H$-positive, and $\varepsilon=-1$ if $(A)$ holds and hence $\mathcal{M}_{0}$ is $H$-negative. Thus,

$$
X^{[*]}=\left[\begin{array}{cc}
X_{11}^{*} & 0 \\
\varepsilon H_{2}^{-1} X_{12}^{*} & H_{2}^{-1} X_{22}^{*} H_{2}
\end{array}\right] .
$$

Furthermore, we have that

$$
X^{[*]} X-X X^{[*]}=\left[\begin{array}{cc}
* & * \\
* & H_{2}^{-1} X_{22}^{*} H_{2} X_{22}-X_{22} H_{2}^{-1} X_{22}^{*} H_{2}+\varepsilon H_{2}^{-1} X_{12}^{*} X_{12}
\end{array}\right] .
$$

Note that by assumption $X_{22}+X_{22}^{[*]}$ has real spectrum. If $(A)$ holds, i.e., $\varepsilon=-1$, then $X_{22}$ is $H_{2}$-hyponormal and, hence, has an invariant maximal $H_{2}$-nonpositive subspace $\mathcal{M}_{1}$ by Theorem 4.3. Thus, $\mathcal{M}_{0} \oplus \mathcal{M}_{1}$ is $X$-invariant and maximal $H$-nonpositive. If, on the other hand, $(B)$ holds, that is, $\varepsilon=+1$, then $X_{22}$ is $\left(-H_{2}\right)$-hyponormal and it has an invariant maximal $\left(-H_{2}\right)$-nonpositive subspace $\mathcal{M}_{1}$ by Theorem 4.3 . Clearly, $\mathcal{M}_{1}$ is then $H_{2}$-nonnegative and $\mathcal{M}_{0} \oplus \mathcal{M}_{1}$ is $X$-invariant and maximal $H$-nonnegative.

Note that the hypothesis (4.1) is guaranteed to hold by the assumption that either $X+X^{[*]}$ or $X-X^{[*]}$ has rank one. However, matrices of this form are $H$-dissipative up to a constant, and for that case the result of the theorem is known; see [13]. Also note that the result of the theorem is not true without any condition, that is, we cannot expect the existence of a maximal $H$-nonnegative $X$-invariant subspace $\mathcal{M}$ containing $\mathcal{M}_{0}$ in general, as shown in the example presented in Section 2. We conclude the paper by providing an example illustrating Theorem 4.4.

EXAMPLE 2. Let

$$
X=\left[\begin{array}{l|ll}
2 & 0 & 2 \\
\hline 0 & 2 & 1 \\
0 & 0 & 0
\end{array}\right], \quad Y=\left[\begin{array}{c|cc}
2 & 4 & -4 \\
\hline 0 & 0 & 2 \\
0 & 0 & 2
\end{array}\right], \quad H=\left[\begin{array}{c|cc}
-1 & 0 & 0 \\
\hline 0 & 0 & -1 \\
0 & -1 & 0
\end{array}\right] .
$$

Then

$$
X^{[*]}=\left[\begin{array}{ccc}
2 & 0 & 0 \\
2 & 0 & 1 \\
0 & 0 & 2
\end{array}\right] \text { and } Y^{[*]}=\left[\begin{array}{ccc}
2 & 0 & 0 \\
-4 & 2 & 2 \\
4 & 0 & 0
\end{array}\right]
$$


Note that $X$ is $H$-normal and that $Y$ is $H$-hyponormal. Consider the $H$-negative subspace $\mathcal{M}_{p}=\operatorname{Span}\left(e_{1}\right)$. Then $\mathcal{M}_{1}$ is invariant for $X$ and $Y$, but neither for $X^{[*]}$ nor $Y^{[*]}$. We now obtain that

$$
\left.X\right|_{\mathcal{M}_{p}^{[\perp]}}+\left(\left.X\right|_{\mathcal{M}_{p}^{[\perp]}}\right)^{[*]_{H_{22}}}=\left[\begin{array}{ll}
2 & 2 \\
0 & 2
\end{array}\right] \quad \text { and }\left.Y\right|_{\mathcal{M}_{p}^{[\perp]}}+\left(\left.Y\right|_{\mathcal{M}_{p}^{[\perp]}}\right)^{[*]_{H_{22}}}=\left[\begin{array}{ll}
2 & 4 \\
0 & 2
\end{array}\right]
$$

and hence, (4.1) is satisfied. Indeed, $\mathcal{M}_{p}$ is contained in the maximal $H$-nonpositive subspace $\mathcal{M}_{1}=\operatorname{Span}\left(e_{1}, e_{2}\right)$ that is invariant for both $X$ and $Y$. Note, that $\mathcal{M}_{1}$ is also invariant for $X^{[*]}$ as it should by Proposition 2.1, but $\mathcal{M}_{1}$ is not invariant for $Y^{[*]}$.

\section{REFERENCES}

[1] T. Ando. Linear Operators in Krein Space. Hokkaido University, Research Institute of Applied Electricity, Division of Applied Mathematics, Sapporo, Japan, 1979.

[2] T. Ya. Azizov and I. S. Iohvidov. Linear Operators in Spaces with an Indefinite Metric. John Wiley and Sons, Ltd., Chichester, 1989. (Translated from Russian.)

[3] I. Gohberg, P. Lancaster, and L. Rodman. Matrices and Indefinite Scalar Products. Birkhäuser Verlag, Basel, Boston, Stuttgart, 1983.

[4] I. Gohberg and B. Reichstein. On classification of normal matrices in an indefinite scalar product. Integral Equations Operator Theory, 13:364-394, 1990.

[5] I. Gohberg and B. Reichstein. On $H$-unitary and block-Toeplitz $H$-normal operators. Linear and Multilinear Algebra, 30:17-48, 1991.

[6] I. Gohberg and B. Reichstein. Classification of block-Toeplitz $H$-normal operators. Linear and Multilinear Algebra, 34:213-245, 1993.

[7] I. S. Iohvidov. On a lemma of Ky Fan generalizing the fixed point principle of A. N. Tihonov. Dokl. Akad. Nauk SSSR, 159:501-504, 1964; English translation: Soviet Math. Dokl. 5:1523-1526, 1964.

[8] I. S. Iohvidov, M. G. Kreĭn, and H. Langer. Introduction to the spectral theory of operators in spaces with an indefinite metric. Mathematical Research 9, Akademie-Verlag, Berlin, 1982.

[9] G. K. Langer. Invariant subspaces of linear operators acting in a space with indefinite metric. Dokl. Akad. Nauk SSSR, 169:12-15, 1966. (Russian.)

[10] H. Langer. Invariante Teilräume definisierbarer $J$-selbstadjungierter Operatoren. Ann. Acad. Sci. Fenn. Ser. A I, 475, 1971. (German.)

[11] H. Langer and F. H. Szafraniec. Bounded normal operators in a Pontryagin space, submitted for publication.

[12] C. V. M. van der Mee, A. C. M. Ran, and L. Rodman. Polar decompositions and related classes of operators in spaces $\Pi_{\kappa}$. Integral Equations and Operator Theory, 44:50-70, 2002.

[13] C. Mehl, A. C. M. Ran, and L. Rodman. Semidefinite invariant subspaces: degenerate inner products. Operator Theory: Advances and Applications, 149:475-494, 2004.

[14] C. Mehl and L. Rodman. Classes of normal matrices in indefinite inner products. Linear Algebra Appl., 336:71-98, 2001.

[15] A. C. M. Ran and D. Temme. Dissipative matrices and invariant maximal semidefinite subspaces. Linear Algebra Appl., 212/213:169-214, 1994. 